\title{
Bilateral corneal ulceration in ocular graft-versus-host disease
}

This article was published in the following Dove Press journal:

Clinical Ophthalmology

30 October 2013

Number of times this article has been viewed

\section{William Stevenson \\ Hasanain Shikari \\ Ujwala S Saboo \\ Francisco Amparo \\ Reza Dana}

Cornea Service, Massachusetts Eye and Ear Infirmary, Boston, MA, USA
Correspondence: Reza Dana

Cornea Service, Massachusetts Eye and Ear Infirmary, 243 Charles Street.

Boston, MA 02II4, USA

$\mathrm{Tel}+\mathrm{I} 6175734439$

Fax+l 6175734300

Email reza_dana@meei.harvard.edu
Purpose: To report on corneal ulceration in ocular graft-versus-host disease (GVHD).

Methods: This was a retrospective, observational case series investigating corneal ulceration and perforation in a cohort of ocular GVHD patients seen between June 2007 and October 2012.

Results: Four of 243 ocular GVHD patients developed corneal ulcerations attributable to ocular GVHD, and all four cases involved bilateral corneal ulceration. The median length of time from the diagnosis of ocular GVHD to the diagnosis of the first corneal ulceration was 317 days (range 168-434). The median length of time between the diagnosis of corneal ulceration in each patient's first and second eye was 248 days (range 9-645). Outcomes varied from complete resolution with medical treatment to corneal perforation necessitating penetrating keratoplasty. In cases of corneal perforation, the median length of time from the diagnosis of corneal ulceration to perforation was 10 days (range $0-20$ ). Common clinical features included: centrally or paracentrally located ulcerations and perforations, concomitant dry eye, and the use of topical or systemic corticosteroids.

Conclusion: Frequent follow-up and bilateral monitoring are highly recommended in cases of ocular GVHD-associated stromal thinning, as bilateral involvement or rapid progression to corneal perforation can occur.

Keywords: graft-versus-host disease, ocular graft-versus-host disease, corneal ulceration, corneal perforation

\section{Introduction}

Allogeneic hematopoietic stem cell transplantation (HSCT) is used to treat a variety of acquired and congenital hematological disorders. ${ }^{1}$ However, allogeneic HSCT can give rise to graft-versus-host disease (GVHD), a significant cause of morbidity and mortality in allogeneic HSCT recipients. ${ }^{2}$ Historically, GVHD has been classified as either acute or chronic based on an arbitrary time point of 100 days posttransplantation. Classification criteria have since been developed that more accurately reflect the signs and symptoms associated with each subtype of GVHD. ${ }^{3}$ Chronic GVHD arises in up to $80 \%$ of allogeneic HSCT recipients who survive beyond 100 days posttransplantation. ${ }^{4}$ Diagnostic or distinctive manifestations of chronic GVHD can occur in the skin, mouth, gastrointestinal tract, genitals, lungs, musculoskeletal system, and eyes. ${ }^{3}$

Ocular manifestations occur in up to $80 \%$ of chronic GVHD patients. ${ }^{5}$ Although chronic GVHD can theoretically affect any ocular structure, it generally targets components of the ocular surface system, including the cornea, conjunctiva, eyelid, lacrimal glands, meibomian glands, and lacrimal drainage system..$^{5-7}$ The distinctive manifestations of ocular GVHD include new-onset dry, gritty, or painful eyes, confluent areas 
of punctate keratopathy, and cicatricial conjunctivitis. ${ }^{3}$ These ocular manifestations have the potential to impact quality of life negatively and hinder the performance of activities of daily living. ${ }^{5,8}$ In extreme cases, ocular GVHD can give rise to sight-threatening complications, such as corneal ulceration and perforation. ${ }^{9}$ Herein, we present four cases of bilateral corneal ulceration associated with ocular GVHD.

\section{Materials and methods}

This was a retrospective, observational case series investigating the occurrence of ocular GVHD-associated corneal ulceration in a cohort of patients seen by the Cornea Service at the Massachusetts Eye and Ear Infirmary in Boston, MA between June 2007 and October 2012. According to the National Institutes of Health, the diagnosis of ocular GVHD requires either new-onset ocular dryness with a mean Schirmer's score of $\leq 5 \mathrm{~mm}$ or new-onset clinical signs of dry eye with a Schirmer's score of 6-10 mm occurring in a patient with manifestations of chronic GVHD in at least one other organ system. ${ }^{3,5}$ The following variables were calculated: 1) the incidence of corneal ulceration secondary to ocular GVHD, 2) the median age at presentation for corneal ulceration, 3 ) the median length of time from HSCT to the diagnosis of ocular GVHD, 4) the median length of time from the diagnosis of ocular GVHD to the diagnosis of the first corneal ulceration, 5) the median length of time between the diagnosis of corneal ulceration in each patient's first and second eye, and 6) the median length of time from corneal ulceration to perforation. Results are presented as the median (range). This study adhered to the principles of the Declaration of Helsinki, and the study protocol was approved by the Institutional Review Board at Massachusetts Eye and Ear Infirmary.

\section{Results}

\section{Patient characteristics}

A total of 243 ocular GVHD patients were seen by the Cornea Service at Massachusetts Eye and Ear Infirmary between June 2007 and October 2012. Four of 243 ocular GVHD patients $(1.6 \%)$ developed corneal ulcerations attributable to ocular GVHD, and all four of these cases involved bilateral corneal ulceration. Patient demographics and clinical characteristics are summarized in Table 1 . The median age was 46 years (range 28-59) at the time of presentation for each patient's first corneal ulceration. The underlying hematological malignancies were varied, including acute lymphoblastic leukemia (ALL), acute myeloid leukemia (AML), chronic myeloid leukemia (CML), and non-Hodgkin's lymphoma (NHL).
The median length of time from HSCT to the diagnosis of ocular GVHD was 446 days (range 115-4897). The median length of time from the diagnosis of ocular GVHD to the diagnosis of the first corneal ulceration was 317 days (range 168-434). The median length of time between the diagnosis of corneal ulceration in the first and second eye was 248 days (range 9-645). Two patients experienced corneal perforations; the median length of time from the diagnosis of corneal ulceration to perforation was 10 days (range $0-20$ ).

\section{Case I}

A 53-year-old man presented complaining of dryness, redness, photophobia, and fluctuating visual acuity that began shortly after he underwent cataract surgery. The patient's medical history was significant for ALL, necessitating allogeneic HSCT that resulted in ocular GVHD. At presentation, ocular examination revealed a $4.5 \times 9.0 \mathrm{~mm}$ inferocentral area of stromal thinning in the patient's left (first) eye. The affected eye was treated with lubricating eye drops, prophylactic antibiotics, corticosteroids, and a sutureless amniotic membrane. However, the corneal ulceration perforated, necessitating penetrating keratoplasty with temporal tarsorrhaphy. The patient returned 1 year later, reporting a decrease in visual acuity, ocular pain, and redness. Ocular examination revealed a $3.5 \times 2.2 \mathrm{~mm}$ paracentral area of stromal thinning in the same (first) eye. The patient was treated with lubricating eye drops, prophylactic antibiotics, corticosteroids, and a therapeutic contact lens. The ulcer resolved over the next several weeks, leaving behind a small scar with thinning and neovascularization. The patient returned several weeks later, complaining of irritation and blurred vision. Ocular examination revealed a $2.2 \mathrm{~mm}^{2}$ area of stromal thinning in the right (second) eye. The patient was treated with lubricating eye drops, prophylactic antibiotics, corticosteroids, and a therapeutic contact lens. The corneal ulceration in the patient's right (second) eye resolved. However, the patient returned with another corneal perforation in his left (first) eye located in an area of residual thinning. Corneal glue was placed, and a therapeutic penetrating keratoplasty was performed. At the patient's last follow-up visit, his best corrected visual acuity (BCVA) was 20/30 in his right eye and 20/400 in his left eye.

\section{Case 2}

A 28-year-old woman presented complaining of dryness, irritation, photophobia, and a "gush" of fluid from her right (first) eye that occurred 5 days before she presented to our clinic. The patient's past medical history was significant for herpes simplex virus keratitis in her right eye, AML treated 
Table I Patient characteristics

\begin{tabular}{|c|c|c|c|c|}
\hline & Case I & Case 2 & Case 3 & Case 4 \\
\hline Age/sex & $53 /$ male & 28/female & $59 /$ female & $39 /$ male \\
\hline Underlying disease & ALL & AML & $\mathrm{CML}$ & $\mathrm{NHL}$ \\
\hline \multirow[t]{2}{*}{ Conditioning regimen } & Myeloablative & Myeloablative & Myeloablative & Nonmyeloablative \\
\hline & Cytoxan/TBI & Cytoxan/TBI & Cytoxan/TBI & fludarabine/busulfan \\
\hline Donor sex/relation & Male/unrelated & Male/unrelated & Male/related & Female/related \\
\hline Donor HLA status & Matched 6/6 & Matched 5/6 & Matched 5/6 & Matched 6/6 \\
\hline Date of HSCT & $12 / 18 / 2008$ & $4 / 4 / 2008$ & $8 / 22 / 1997$ & $2 / 15 / 2006$ \\
\hline Transplant type & PBSCT & PBSCT & BMT & PBSCT \\
\hline GVHD prophylaxis & $\begin{array}{l}\text { Tacrolimus, } \\
\text { sirolimus }\end{array}$ & $\begin{array}{l}\text { Prednisolone, } \\
\text { tacrolimus, sirolimus }\end{array}$ & Cyclosporine & Cyclosporine \\
\hline GVHD manifestations & Skin, liver, eyes & Liver, lungs, eyes & Eyes & Skin, liver, eyes \\
\hline Days from HSCT to ocular & 447 & 445 & 4,897 & 115 \\
\hline \multicolumn{5}{|l|}{ GVHD diagnosis } \\
\hline \multirow[t]{2}{*}{ Days from HSCT to ulceration } & OD: I,287 & OD: 670 & OD: 5,065 & OD: 1,910 \\
\hline & OS: 881 & OS: I,315 & OS: 5,155 & OS: 1,919 \\
\hline Days from HSCT to perforation & OS: 901 & OD: 670 & NA & NA \\
\hline Culture results & Staphylococcus aureus & Hemolytic streptococci & NA & NA \\
\hline \multirow[t]{2}{*}{ Visual acuity at presentation } & OD: $20 / 100$ & OD: $20 / 200$ & OD: $20 / 40$ & OD: $20 / 400$ \\
\hline & OS: CF@ I foot & OS: $20 / 300$ & OS: $20 / 100$ & OS: $20 / 200$ \\
\hline Visual acuity at last & OD: $20 / 30$ & OD: $20 / 30$ & OD: $20 / 30$ & OD: hand motion \\
\hline follow-up visit & OS: $20 / 400$ & OS: $20 / 30$ & OS: $20 / 30$ & OS: $20 / 80$ \\
\hline Duration of topical & Unknown & OD: 2 weeks & OD: NA & NA \\
\hline corticosteroids before ulceration & & OS: 3 weeks & OS: I week & \\
\hline $\begin{array}{l}\text { Duration of oral corticosteroids } \\
\text { before ulceration }\end{array}$ & 2 months & 15 months & NA & 60 months \\
\hline
\end{tabular}

Abbreviations: ALL, acute lymphoblastic leukemia; AML, acute myeloid leukemia; NHL, non-Hodgkin's lymphoma; CML, chronic myeloid leukemia; TBI, total body irradiation; HLA, human leukocyte antigen; HSCT, hematopoietic stem cell transplantation; PBSCT, peripheral blood stem cell transplantation; BMT, bone marrow transplantation; GVHD, graft-versus-host disease; OD, right eye; OS, left eye; CF, count fingers; NA, not applicable.

with allogeneic HSCT, and ocular GVHD. At presentation, ocular examination revealed an inferocentral corneal perforation plugged with iris tissue in the patient's right (first) eye. Given that the anterior chamber was quiet, deep, and intact, surgery was deferred and aggressive broad-spectrum antimicrobial therapy with lubrication was initiated. The corneal perforation eventually healed, leaving behind a small scar with an adherent leukoma. The patient subsequently underwent cataract surgery in her left eye; the postoperative course was uneventful until 1 month after surgery, when she returned with pain, redness, and decreased vision. Ocular examination revealed a $2.0 \times 1.2 \mathrm{~mm}$ inferocentral area of stromal thinning with underlying infiltrate and a $<1.0 \mathrm{~mm}^{2}$ superocentral area of stromal thinning in the patient's left (second) eye. The patient was treated with broad-spectrum antimicrobial therapy and aggressive lubrication, after which the ulcers healed with some residual thinning and scarring. At the patient's last follow-up visit, her BCVA was 20/30 in her right eye and 20/30 in her left eye.

\section{Case 3}

A 59-year-old woman presented complaining of dryness and irritation. The patient's past medical history was significant for CML treated with allogeneic HSCT that resulted in ocular GVHD. Ocular examination revealed a $1.0 \times 1.1 \mathrm{~mm}$ inferonasal epithelial defect with stromal thinning without any signs of infection in her right (first) eye. The patient was treated with lubricating eye drops, prophylactic antibiotics, corticosteroids, and a therapeutic contact lens. The corneal ulceration healed over the next several months, leaving behind minimal residual thinning. Shortly thereafter, the patient returned with a $1 \mathrm{~mm}^{2}$ area of stromal thinning in her left (second) eye. In addition to the standard therapy, she was prescribed topical anakinra (Kineret ${ }^{\circledR}$; Swedish Orphan Biovitrum, Stockholm, Sweden), and her signs and symptoms resolved with minimal residual thinning. The patient's BCVA was 20/30 in her right eye and 20/30 in her left eye at her last follow-up visit.

\section{Case 4}

A 39-year-old man presented complaining of blurred vision and photophobia. The patient's past medical history was significant for NHL treated with allogeneic HSCT that resulted in ocular GVHD, and an episode of stromal thinning. The patient presented complaining of blurred vision without any associated pain. Ocular examination revealed a $2 \mathrm{~mm}^{2}$ area of 
stromal thinning in the patient's right (first) eye. Lubricating eye drops and cyclosporine (Restasis ${ }^{\circledR}$; Allergan, Irvine, CA, USA) were increased, prophylactic antibiotics were initiated, and a therapeutic contact lens was placed. Shortly after this visit, the patient was admitted to Massachusetts General Hospital for systemic manifestations of chronic GVHD. At the time of admittance, the patient complained of severe pain, decreased visual acuity, and photophobia. Ocular examination revealed a $3.6 \times 4.4 \mathrm{~mm}$ inferocentral area of stromal thinning in the patient's right (first) eye. The corneal thinning continued to increase in the patient's right (first) eye, and a central epithelial defect began to form in his left (second) eye. The epithelial defect in the left (second) eye rapidly progressed to stromal melting, necessitating treatment with a scleral lens. Following placement of the scleral lens, the patient reported increased comfort and his BCVA improved to 20/80 in his left eye; however, he died several days later from complications of systemic GVHD.

\section{Discussion}

The mechanisms underlying corneal ulceration and perforation in ocular GVHD are incompletely understood. GVHD occurs when donor-derived immune cells recognize and attack recipient tissues. ${ }^{10}$ Donor-derived $\mathrm{T}$ cells and innate immune cells have been identified in ocular specimens obtained after allogeneic HSCT, and T cells predominate in the inflammatory lesions associated with ocular GVHD. ${ }^{1-13}$ T-cell-associated cytokines and chemokines have been identified in the tears and ocular surface of ocular GVHD patients. ${ }^{14-16}$ Perforated corneal specimens from patients with chronic GVHD have been shown to contain $\mathrm{T}$ cells and macrophages, apoptotic epithelial cells and keratocytes, and intense expression of matrix metalloproteinase $9 .{ }^{17-20}$ Pretreatment regimens that involve radiation or chemotherapy can damage ocular tissues, and ocular GVHD has been associated with epithelial-mesenchymal transition and fibrosis. ${ }^{21,22}$ Dry eye is the most common ocular manifestation of chronic GVHD, and severe dry eye has been associated with corneal epitheliopathy, ulceration, and perforation. , $23,24^{2}$ Chronic inflammation-induced corneal neuropathy or limbal stem cell deficiency may also contribute to corneal ulceration and perforation in ocular GVHD. ${ }^{17-20,25,26}$

Four of 243 ocular GVHD patients developed corneal ulcerations attributable to ocular GVHD, yielding an incidence of $1.6 \%$ in this patient population. Given that Massachusetts Eye and Ear Infirmary is a tertiary referral center it is unclear how representative this patient population is of the ocular GVHD population at large. The cases presented herein share a number of similarities that may provide insight into the pathogenesis of corneal ulceration in ocular GVHD. For example, bilateral involvement occurred in all of the cases, highlighting the systemic immune etiology of GVHD. Bilateral corneal ulcers have been described in a variety of immune-mediated disorders, including Sjögren's syndrome and rheumatoid arthritis. ${ }^{27,28}$ Severe dry eye was present in all of the cases, and severe dry eye has been associated with corneal ulceration and perforation. The corneal ulcerations observed in the present study occurred in central or paracentral locations, suggestive of neurotrophic ulceration or limbal stem cell deficiency. Unsurprisingly, all of the cases involved the use of topical or systemic corticosteroids, either prior to or at the time of corneal ulceration. Corticosteroids, particularly topical corticosteroids, have been linked to corneal thinning, ulceration, and perforation. ${ }^{27}$ However, corticosteroids are frequently used to promote quiescence in cases of severe ocular surface inflammation; hence, corticosteroid use may be a marker of heightened disease severity.

Despite therapeutic intervention, two of the corneal ulcerations progressed to perforation, and all four cases involved bilateral corneal ulceration. These findings suggest that frequent follow-up and bilateral ocular examinations are essential, as bilateral involvement or rapid progression to corneal perforation can occur. Although ocular GVHD undoubtedly contributed to all of the cases presented herein, the pathogenesis of these corneal ulcerations and perforations was likely multifactorial. For example, several of the patients underwent cataract surgery immediately before they developed corneal ulcerations, suggesting that the trauma and inflammation associated with ocular surgery may predispose these patients to developing corneal ulcerations. Corticosteroids should be used judiciously; while corticosteroids can decrease ocular surface inflammation, they may also increase the risk of corneal ulceration or perforation. Topical cyclosporine has proven beneficial in the treatment of ocular GVHD-associated dry eye, and cyclosporine is currently being investigated as a prophylactic treatment for the prevention of ocular GVHD. ${ }^{29,30}$ Topical interleukin 1 inhibition has shown promise in the treatment of dry eye disease; however, further study is needed before this can be routinely recommended for ocular GVHD. ${ }^{31}$ Cases of ocular GVHD-associated corneal ulceration should be treated with prophylactic antibiotics, and cultures should be performed as needed to rule out infection. Corneal ulcerations may also benefit from the use of aggressive lubrication and therapeutic contact lenses. ${ }^{5,6}$ 
The surgical management of a corneal perforation is largely dictated by the size of the perforation and the presence or absence of anterior-chamber leakage. Corneal transplantation in the setting of ocular GVHD is considered high-risk because of the intense inflammation at the ocular surface. A small perforation may benefit from the use of a bandage contact lens, cyanoacrylate tissue adhesive, or multilayer amniotic membrane transplantation; penetrating keratoplasty is generally deferred until the eye is quiet, as the benefits may be offset by the increased risk of graft failure. ${ }^{32,33}$ A large perforation, however, often requires full-thickness tectonic corneal grafting. Further investigation will be necessary to elucidate fully the pathogenesis of ocular GVHD, improve diagnostic and grading criteria, identify risk factors for corneal ulceration and perforation, and provide evidenced-based treatment guidelines.

\section{Disclosure}

The authors report no conflicts of interest in this work.

\section{References}

1. Li HW, Sykes M. Emerging concepts in haematopoietic cell transplantation. Nat Rev Immunol. 2012;12(6):403-416.

2. Lee SJ, Flowers ME. Recognizing and managing chronic graftversus-host disease. Hematology Am Soc Hematol Educ Program. 2008:134-141.

3. Filipovich AH, Weisdorf D, Pavletic S, et al. National Institutes of Health consensus development project on criteria for clinical trials in chronic graft-versus-host disease: I. Diagnosis and staging working group report. Biol Blood Marrow Transplant. 2005;11(12): 945-956.

4. Przepiorka D, Anderlini P, Saliba R, et al. Chronic graft-versushost disease after allogeneic blood stem cell transplantation. Blood. 2001;98(6):1695-1700.

5. Dietrich-Ntoukas T, Cursiefen C, Westekemper H, et al. Diagnosis and treatment of ocular chronic graft-versus-host disease: report from the German-Austrian-Swiss Consensus Conference on Clinical Practice in chronic GVHD. Cornea. 2012;31(3):299-310.

6. Kim SK. Update on ocular graft versus host disease. Curr Opin Ophthalmol. 2006;17(4):344-348

7. Gipson IK. The ocular surface: the challenge to enable and protect vision: the Friedenwald lecture. Invest Ophthalmol Vis Sci. 2007;48(10):4390; 4391-4398.

8. Westeneng AC, Hettinga Y, Lokhorst H, Verdonck L, van Dorp S, Rothova A. Ocular graft-versus-host disease after allogeneic stem cell transplantation. Cornea. 2010;29(7):758-763.

9. Hessen M, Akpek EK. Ocular graft-versus-host disease. Curr Opin Allergy Clin Immunol. 2012;12(5):540-547.

10. Blazar BR, Murphy WJ, Abedi M. Advances in graft-versus-host disease biology and therapy. Nat Rev Immunol. 2012;12(6):443-458.

11. Saito T, Shinagawa K, Takenaka K, et al. Ocular manifestation of acute graft-versus-host disease after allogeneic peripheral blood stem cell transplantation. Int J Hematol. 2002;75(3):332-334.

12. Fei WL, Chen JQ, Du X, et al. Dry eye in graft-versus-host disease. Zhonghua Yan Ke Za Zhi. 2003;39(11):686-690. Chinese.
13. Rojas B, Cuhna R, Zafirakis P, et al. Cell populations and adhesion molecules expression in conjunctiva before and after bone marrow transplantation. Exp Eye Res. 2005;81(3):313-325.

14. Riemens A, Stoyanova E, Rothova A, Kuiper J. Cytokines in tear fluid of patients with ocular graft-versus-host disease after allogeneic stem cell transplantation. Mol Vis. 2012;18:797-802.

15. Kang MH, Kim MK, Lee HJ, Lee HI, Wee WR, Lee JH. Interleukin-17 in various ocular surface inflammatory diseases. $J$ Korean Med Sci. 2011;26(7):938-944.

16. Westekemper H, Meller S, Citak S, et al. Differential chemokine expression in chronic GVHD of the conjunctiva. Bone Marrow Transplant. 2010;45(8):1340-1346.

17. Yeh PT, Hou YC, Lin WC, Wang IJ, Hu FR. Recurrent corneal perforation and acute calcareous corneal degeneration in chronic graft-versushost disease. J Formos Med Assoc. 2006;105(4):334-339.

18. Suzuki M, Usui T, Kinoshita N, Yamagami S, Amano S. A case of sterile corneal perforation after bone marrow transplantation. Eye (Lond). 2007;21(1):114-116.

19. Inagaki E, Ogawa Y, Matsumoto Y, Kawakita T, Shimmura S, Tsubota K. Four cases of corneal perforation in patients with chronic graft-versushost disease. Mol Vis. 2011;17:598-606.

20. Yoshida A, Kawano Y, Kato K, et al. Apoptosis in perforated cornea of a patient with graft-versus-host disease. Can J Ophthalmol. 2006;41(4):472-475.

21. Copelan EA. Hematopoietic stem-cell transplantation. $N$ Engl J Med. 2006;354(17):1813-1826.

22. Ogawa Y, Shimmura S, Kawakita T, Yoshida S, Kawakami Y, Tsubota K. Epithelial mesenchymal transition in human ocular chronic graft-versus-host disease. Am J Pathol. 2009;175(6):2372-2381.

23. Wang Y, Ogawa Y, Dogru M, et al. Baseline profiles of ocular surface and tear dynamics after allogeneic hematopoietic stem cell transplantation in patients with or without chronic GVHD-related dry eye. Bone Marrow Transplant. 2010;45(6):1077-1083.

24. Balaram M, Rashid S, Dana R. Chronic ocular surface disease after allogeneic bone marrow transplantation. Ocul Surf. 2005;3(4):203-211.

25. Lavid FJ, Herreras JM, Calonge M, Saornil MA, Aguirre C. Calcareous corneal degeneration: report of two cases. Cornea. 1995;14(1): 97-102.

26. Adrean SD, Puklin JE. Perforated corneal ulcer with subsequent endophthalmitis in a patient with graft-versus-host disease. Cornea. 2007;26(1):107-108.

27. Pfister RR, Murphy GE. Corneal ulceration and perforation associated with Sjögren's syndrome. Arch Ophthalmol. 1980;98(1):89-94.

28. Kervick GN, Pflugfelder SC, Haimovici R, Brown H, Tozman E, Yee R. Paracentral rheumatoid corneal ulceration. Clinical features and cyclosporine therapy. Ophthalmology. 1992;99(1):80-88.

29. Sall K, Stevenson OD, Mundorf TK, Reis BL. Two multicenter, randomized studies of the efficacy and safety of cyclosporine ophthalmic emulsion in moderate to severe dry eye disease. CsA Phase 3 Study Group. Ophthalmology. 2000;107(4):631-639.

30. Rao SN, Rao RD. Efficacy of topical cyclosporine $0.05 \%$ in the treatment of dry eye associated with graft versus host disease. Cornea. 2006;25(6):674-678.

31. Amparo F, Dastjerdi MH, Okanobo A, et al. Topical interleukin 1 receptor antagonist for treatment of dry eye disease. A randomized clinical trial. JAMA Ophthalmol. 2013;131(6):715-723.

32. Setlik DE, Seldomridge DL, Adelman RA, Semchyshyn TM, Afshari NA. The effectiveness of isobutyl cyanoacrylate tissue adhesive for the treatment of corneal perforations. Am J Ophthalmol. 2005;140(5):920-921.

33. Rodríguez-Ares MT, Touriño R, López-Valladares MJ, Gude F. Multilayer amniotic membrane transplantation in the treatment of corneal perforations. Cornea. 2004;23(6):577-583. 


\section{Publish your work in this journal}

Clinical Ophthalmology is an international, peer-reviewed journal covering all subspecialties within ophthalmology. Key topics include: Optometry; Visual science; Pharmacology and drug therapy in eye diseases; Basic Sciences; Primary and Secondary eye care; Patient Safety and Quality of Care Improvements. This journal is indexed on
PubMed Central and CAS, and is the official journal of The Society of Clinical Ophthalmology (SCO). The manuscript management system is completely online and includes a very quick and fair peer-review system, which is all easy to use. Visit http://www.dovepress.com/ testimonials.php to read real quotes from published authors. 